\title{
Genetic mechanisms of resistance to golden potato cyst nematode Globodera rostochiensis in Solanum phureja
}

\author{
A.A. Egorova ${ }^{1,2 *}$, N.A. Shmakov ${ }^{1}$, S.V. Gerasimova ${ }^{1}$, G.V. Vasilyev ${ }^{1}$, \\ N.V. Shatskaya ${ }^{1}$, K.V. Strygina ${ }^{1}$, D.A. Afonnikov ${ }^{1}$, A.V. Kochetov ${ }^{1,2}$ \\ ${ }^{1}$ Institute of Cytology and Genetics SB RAS, Novosibirsk, Russia \\ ${ }^{2}$ Novosibirsk StateUniversity, Novosibirsk, Russia \\ *e-mail:egorova@bionet.nsc.ru
}

Key words: potato, RNA-seq, Solanum phureja, resistance, Globodera rostochiensis

Motivation and Aim: Golden potato cyst nematode (GPCN) is an important pathogen of potatoes, tomatoes, and other plants in the family Solanaceae. GPCN juveniles damages the roots of susceptible plants. Nowadays GPCN is found worldwide and it appears to be the one of the most deleterious pathogens for potato. Protection against GPCN is complicated because the eggs of nematodes can remain viable in the soil for more than 30 years. In addition, most chemical nematicides are not efficient, thus the protection is mostly obtained through the introduction of the resistance genes.

In this study we analyzed the resistant cultivar of diploid potato Solanum phureja to reveal new resistance genes through comparison of root transcriptomes of resistant and susceptible genotypes.

Methods and Algorithms: For RNA-seq, total RNA was extracted from root samples collected in time points 0,24 and 72 hours after inoculation with GPCN. Sample preparation was carried out by colleagues from Vavilov Institute of Plant Genetic Resources and All Russian Research Institute for Plant Protection (Saint Petersburg, Russia) according to [1]. Sequencing was performed on Illumina NextSeq 500 platform. FastQC and Prinseq tools were used to assess sequences quality and filter the libraries. STAR and TopHat were used to map the filtered libraries to the reference genome. Search for the differentially expressed genes was performed using Cufflinks pipeline and EdgeR package for R. Lists of differentially expressed genes (DEGs) were further analyzed with Biomart and the databases AgriGO, KEGG, and PlantCyc. De novo transcriptome assembling was carried out with Trinity software. Prediction of NBS-LRR genes was based on their typical domain structure.

Results: Analysis of $S$. phureja transcriptomic data revealed differential expression of a number of genes. According to the literature data, the most probable candidate gene(s) for resistance against GPCN may belong to the NBS-LRR family. These receptors recognize specific molecular patterns and can induce programmed cell death at the site of a pathogen invasion. Thus, we focused our analysis on this type of R-genes. Candidate resistance genes expressed in $S$. phureja resistant cultivar were predicted for further analysis of segregating populations.

Conclusion: Candidate pathogen major resistance genes were predicted by transcriptome analysis of two $S$. phureja genotypes contrasting in GPCN resistance.

Acknowledgements: Supported by grant from RSF No. 16-16-04073.

\section{References}

1. Kochetov A.V. et al. (2017) Differential expression of NBS-LRR-encoding genes in the root transcriptomes of two Solanum phureja genotypes with contrasting resistance to Globodera rostochiensis. BMC Plant Biol. 17(Suppl 2):251. 